\title{
Filtro de vena cava y embarazo: algunas certezas y muchas incertidumbres
}

\author{
Vena cava filter and pregnancy: some certainties and many doubts
}

\author{
José Antonio Díaz Peromingo 1 , Manuel José Núñez Fernández² \\ ${ }^{1}$ Complejo Hospitalario Universitario de Santiago. ${ }^{2}$ Complejo Hospitalario Universitario de Pontevedra. España.
}

\section{RESUMEN}

El embarazo constituye un estado protrombótico durante el cual se producen en la mujer cambios anatómicos y fisiológicos que conllevan un incremento en la incidencia de fenómenos tromboembólicos. Así, las mujeres embarazadas tienen un riesgo 5 veces mayor de padecer un evento tromboembólico que las no embarazadas. La enfermedad tromboembólica venosa es una causa importante de morbilidad y mortalidad maternas y la embolia pulmonar es la causa más común de mortalidad materna en los países desarrollados. En el presente trabajo se revisan aspectos como la valoración de riesgo tromboembólico en la mujer embarazada, el diagnóstico, profilaxis y tratamiento, con especial atención al uso del filtro de vena cava en mujeres embarazadas en términos de indicaciones, colocación, efectividad, complicaciones y riesgos tanto para la madre como para el feto. Palabras clave: filtro de vena cava, embarazo, profilaxis, tratamiento

\section{INTRODUCCIÓN}

El embarazo constituye de por sí un estado protrombótico. Durante el embarazo se producen en la mujer cambios anatómicos y fisiológicos que van desde adaptaciones del sistema hemostático al momento del parto (hipercoagulabilidad para minimizar la hemorragia propia del parto), el estasis venoso causado por el aumento de tamaño del útero (se reduce en casi un 50\% la velocidad del flujo venoso en las extremidades inferiores hacia la mitad de la gestación), compresión por parte del útero de estructuras como la propia vena cava inferior y daño endotelial causado por la propia distensión ${ }^{1}$. Estos cambios conllevan un incremento en la incidencia de fenómenos tromboembólicos con una frecuencia que varía entre 0,76 y 1,72 casos por 1000 embarazos $^{2}$.

Las mujeres embarazadas tienen un riesgo 5 veces mayor de padecer un evento tromboembólico que las no embarazadas ${ }^{3,4}$. La probabilidad de tener una trombosis es similar en los tres trimestres del embarazo y también está aumentada en las 6 semanas siguientes al parto ${ }^{5}$. La enfermedad tromboembólica venosa (ETV) es causa importante de morbilidad y mortalidad maternas ${ }^{6-8}$. El miembro inferior izquierdo es el que más frecuentemente se ve afectado por la trombosis en las mujeres gestantes $(82 \%)^{2,9}$. La embolia pulmonar constituye la causa más común de mortalidad materna en los países desarrollados ${ }^{3,4,10}$.

\section{VALORACIÓN DEL RIESGO Y PROFILAXIS}

La hipercoagulabilidad que se produce durante el embarazo es el factor de riesgo más importante para el desarrollo de trombosis. Aumenta la síntesis de fibrina, disminuye la actividad fibrinolítica, aumentan los niveles de los factores II, VII, VIII y X de la coagulación, disminuyen progresivamente los niveles de proteína S y aumenta la resistencia adquirida a la proteína $\mathrm{C}$ activada. Todos estos cambios reflejan la adaptación fisiológica a los cambios hemostáticos que se van a producir en el momento del parto ${ }^{11,12}$.

El riesgo diario de padecer un evento protrombótico es entre 5 y 10 veces en el preparto y entre 15 y 35 veces en el postparto ${ }^{13}$.

\section{ABSTRACT}

Pregnancy constitutes a prothrombotic state during which anatomical and physiological changes occur in women. This situation leads to an increase in the incidence of thromboembolic phenomena. Thus, pregnant women have a 5 times increased risk of suffering a thromboembolic event than non-pregnant women. Venous thromboembolic disease is a major cause of maternal morbidity and mortality, and pulmonary embolism is the most common cause of maternal mortality in developed countries. In the present work, aspects such as the assessment of thromboembolic risk in pregnant women, diagnosis, prophylaxis and treatment are reviewed, focusing on the use of the vena cava filter in pregnant women in terms of indications, placement, effectiveness, complications and risks for both mother and fetus. Keywords: vena cava filter, pregnancy, profilaxys, treatment

Los factores de riesgo específicos para el desarrollo de eventos tromboembólicos durante el embarazo se muestran en la Tabla 1. En estos casos se debe valorar la administración de profilaxis tromboembólica.

Tabla 1: Factores predisponentes para el desarrollo de fenómenos trombóticos asociados el embarazo.

\begin{tabular}{|l|}
\hline Edad superior a 35 años \\
\hline Realización de cesárea \\
\hline Obesidad \\
\hline Trombofilia \\
\hline Multiparidad \\
\hline Sangrado excesivo \\
\hline Presencia de infecciones o patologías inflamatorias, sepsis puerperal \\
\hline Preeclampsia \\
\hline Inmovilidad \\
\hline Diabetes mellitus \\
\hline Conectivopatías \\
\hline Antecedentes personales o familiares de trombosis \\
\hline Tabaco \\
\hline Síndrome de hiperestimulación ovárica \\
\hline Fertilización in vitro \\
\hline Enfermedad cardiaca \\
\hline Anemia de células falciformes \\
\hline
\end{tabular}

En el caso de la existencia de una trombofilia de alto riesgo, el manejo de ésta durante el embarazo implica el uso de dosis terapéuticas o profilácticas de heparina por el alto riesgo de presentar 
episodios de trombosis venosa profunda o embolia pulmonar22. Aproximadamente el $50 \%$ de las trombosis venosas gestacionales están asociadas con trombofilias hereditarias ${ }^{23}$.

Determinar qué pacientes deben recibir profilaxis no es fácil, si bien parece que una historia de trombofilia hereditaria o adquirida, así como haber tenido una trombosis venosa profunda previa constituyen los factores de riesgo más importantes ${ }^{24}$. Actualmente se recomienda iniciar profilaxis con HBPM en mujeres gestantes con historia previa de tromboembolismo venoso y trombofilia o trombosis venosa múltiple previa (2 o más episodios). En pacientes con una única trombosis previa, sin trombofilia asociada se recomienda seguimiento estricto durante el embarazo y profilaxis en el postparto. En embarazadas con trombofilia conocida, pero sin eventos trombóticos previos, se recomienda seguimiento estricto con valoración del riesgo adicional y profilaxis en el preparto y postparto. No hay consenso en cuanto a dosis adecuada ${ }^{25}$. La profilaxis debe de interrumpirse una vez se ha establecido el trabajo del parto.

En la actualidad, una de las escalas más usadas es la escala de valoración de riesgo tromboembólico de Lyon (Tabla 2). Permite una valoración de la necesidad de profilaxis tromboembólica en el preparto en mujeres con alto riesgo. Valora el riesgo de eventos trombóticos durante el embarazo en base a los antecedentes de enfermedad tromboembólica, presencia de trombofilia y factores de riesgo directamente asociados con la gestación ${ }^{26}$.

\section{MANEJO DE LA TROMBOSIS ASOCIDA AL EMBARAZO}

Actualmente, la administración de heparinas de bajo peso molecular (HBPM) a dosis anticoagulantes, repartida en una o dos dosis diarias, es el método de elección ya que estos fármacos no atraviesan la barrera placentaria y tienen menos efectos adversos (fundamentalmente osteoporosis y trombocitopenia) en comparación con la heparina no fraccionada ${ }^{27,28}$. Si bien, la duración del tratamiento de la enfermedad tromboembólica no está bien definida durante el embarazo, se acepta que sea de al menos 6 meses incluyendo las 6 semanas siguientes al parto ${ }^{29}$. Las HBPM tienen el inconveniente de presentar una vida media más larga que la heparina no fraccionada, lo que puede representar un problema en el momento del parto. La heparina no fraccionada se prefiere en pacientes con insuficiencia renal grave ya que las HBPM se excretan fundamentalmente por el riñón y pueden acumularse si existe insuficiencia renal asociada ${ }^{25}$. La warfarina o el acenocumarol no se usan en el embarazo ya que, al cruzar la barrera placentaria, pueden causar complicaciones en el feto incluyendo malformaciones y muerte ${ }^{29}$. Los anticoagulantes orales de acción directa también cruzan la barrera placentaria y sus efectos en la reproducción humana son desconocidos por lo que están actualmente contraindicados en el embarazo ${ }^{25}$.

En algunos casos como en mujeres donde el evento tromboembólico se diagnostica en el periodo pretérmino (a partir de la semana 37 de gestación) o durante el manejo periparto en el caso de la existencia de amenaza para el feto, el tratamiento anticoagulante puede suponer un riesgo adicional. En estos casos, el uso de filtros de vena cava (FVC) puede evitar la aparición de una embolia pulmonar ${ }^{30-33}$. Una de las indicaciones más aceptadas es la existencia de una trombosis venosa profunda extensa durante el embarazo, con alto riesgo de embolia pulmonar durante el parto y donde la anticoagulación no se puede interrumpir, sobre todo si el trombo está en el eje ilio-femoral y el parto se espera en las próximas 2-3 semanas $^{14-21}$. Sin embargo, la experiencia en el uso de FVC en mujeres embarazadas es limitada. Una revisión sistemática sugiere que pueden ser eficaces en el embarazo con unas tasas de complicaciones similares a las mujeres no embarazadas, pero, al tratarse de una revisión de casos aislados, deben tenerse en cuenta sesgos de publicación ${ }^{35}$.

Tabla 2. Escala de Lyon de valoración de riesgo tromboembólico asociado al embarazo. Una puntuación < 3, no necesidad de profilaxis preparto; 3-5, HBPM comenzando en el tercer trimestre; $\geq 6$, HBPM comenzando en el primer trimestre.

\begin{tabular}{|c|c|c|}
\hline \multicolumn{2}{|c|}{ FACTOR DE RISGO DE ENFERMEDAD TROMBOEMBÓLICA (ETV) } & \multirow{2}{*}{$\frac{\text { PUNTOS }}{6}$} \\
\hline ANTECEDENES PERSONALES & $\begin{array}{l}\text { ETV RELACIONADA CON EL EMBARAZO (PREPARTO), TROMBOSIS VENOSA } \\
\text { CEREBRAL O TVP/EP EN LA INFANCIA }\end{array}$ & \\
\hline & EP o TVP espontánea o inducida por estrógenos & 3 \\
\hline & EP o TVP inducida por factor de riesgo transitorio & 2 \\
\hline & TVP distal espontánea 0 inducida por estrógenos & 2 \\
\hline & TVP distal inducida por factor de riesgo transitorio & 1 \\
\hline \multirow[t]{3}{*}{ Si existe historia personal de ETE } & ETV recurrente & 3 \\
\hline & Trombo residual con signos clínicos de síndrome postrombótico & 3 \\
\hline & ETV en los últimos 2 años & 2 \\
\hline \multirow[t]{2}{*}{ Trombofilia } & $\begin{array}{l}\text { Mutaciones homocigotas, combinado de factores de riesgo de trombofilia, déficit } \\
\text { de proteína C, déficit de proteína S, heterocigosis para la mutación F5 G1691 A } \\
\text { heterocigosis para la mutación F5 G20210 A }\end{array}$ & \\
\hline & No datos de trombofilia, pero sí historia familiar de ETV recurrente y grave & 1 \\
\hline \multirow[t]{4}{*}{ Otros factores de riesgo } & Encamamiento, inmovilización & 2 \\
\hline & Embarazo gemelar & 1 \\
\hline & Edad mayor de 35 años & 1 \\
\hline & IMC mayor de 30 & 1 \\
\hline
\end{tabular}

TVP: trombosis venosa profunda; EP: embolia pulmonar; IMC: índice de masa corporal. HBPM: heparina de bajo peso molecular. 


\section{DIAGNÓSTICO DE ENFERMEDAD TROMBOEMBÓLICA EN EL EMBARAZO}

La trombosis venosa es más frecuente y difícil de diagnosticar en las pacientes embarazadas que en las que no lo están. La presentación más frecuente en la trombosis venosa profunda (TVP) es la hinchazón (88\% de mujeres embarazadas; $79 \%$ en el postparto) y el dolor (79\% de mujeres embarazadas; $95 \%$ en el postparto). Se asocia también dificultar para la deambulación (21\% de mujeres embarazadas; $32 \%$ en el postparto). El eritema es referido en el $26 \%$ en ambos grupos ${ }^{2}$. La trombosis venosa iliaca aislada en más frecuente durante el embarazo y puede manifestarse como dolor abdominal, en la espalda o hinchazón de toda una extremidad inferior. Estos síntomas pueden no ser fáciles de diferenciar de los de la incomodidad propia que acompaña al embarazo normal. Además, el estasis e hinchazón de los miembros inferiores puede también deberse a compresión mecánica por parte del útero agrandado, de los vasos linfáticos y de las estructuras venosas. Así pues, el edema y el malestar en la pierna, abdomen o la espalda, no son síntomas que ayuden al clínico a sospechar de forma clara una trombosis subyacente. Con frecuencia la trombosis no se hace evidente hasta que progresa distalmente en las venas femorales causando dolor y edema en toda la pierna afectada.

Debido al incremento en la actividad fibrinolítica en el estado de hipercoagulabilidad asociado al embarazo, los niveles de dímero D (DD) están elevados por lo que tampoco ayudan en el cálculo de probabilidad diagnóstica'2.

Dado que existe la posibilidad de muerte súbita en mujeres embarazadas por embolia pulmonar (EP), se debe hacer una valoración rápida de la posibilidad de que exista una ETV subyacente cuando se sospeche. Es más, si no hay contraindicación, se debe iniciar anticoagulación antes de establecer el diagnóstico, si la sospecha clínica es muy elevada ${ }^{3}$.

Para confirmar el diagnóstico con técnicas de imagen es preferible usar las menos invasivas y sin radiación asociada si es posible. En el diagnóstico de la TVP en miembros inferiores se usa la ecografía Doppler por compresión venosa. En los casos de trombosis venosa intraabdominal la ecografía tiene una sensibilidad del 42\% en comparación con la resonancia magnética nuclear (RMN) que se sitúa en el 98\%, lo que hace de ésta última la técnica de elección en casos de sospecha de trombosis de vena iliaca, de vena cava 0 vena mesentérica ${ }^{34}$. La tomografía axial computarizada (TAC) puede usarse cuando no hay disponibilidad de RMN en el diagnóstico de TVP intraabdominal pero no es lo ideal por la exposición a la radiación que implica tanto para la madre como para el feto. En cuanto al diagnóstico de EP, la actualización de las Guías de la European Society of Cardiology de 2019 valoran el uso del DD y factores clínicos para diagnosticar o excluir el diagnóstico de una EP durante el embarazo o en el postparto. Así, la negatividad del DD tiene una significación clínica similar a la de las pacientes no embarazadas, evitando la realización de estudios que irradien innecesariamente a la paciente embarazada. En mujeres con sospecha de EP y radiografía de tórax normal, estaría indicada la realización de una gammagrafía pulmonar de ventilación/perfusión; si ésta no fuese accesible, o la radiografía de tórax fuese normal, se debería considerar la realización de una angioTAC pulmonar ${ }^{37}$.

Dos estudios recientes han incidido en cómo mejorar el diagnóstico de EP en mujeres embarazadas, con el objetivo de disminuir la realización de pruebas de imagen potencialmente dañinas, tanto para la madre como para el feto. En el primero se incluyeron 395 mujeres en las que se excluyó la posibilidad de EP mediante el uso de la escala de Ginebra y si presentaban un DD $<0.5 \mu \mathrm{g} / \mathrm{m}^{38}$. El segundo estudio, ARTEMIS, se basó en la adaptación del algoritmo YEARS (signos clínicos de TVP, hemoptisis, diagnóstico de EP como el más probable y DD) usado en mujeres no embarazadas ${ }^{39}$, a mujeres embarazadas con sospecha de EP. La presencia de EP se podría excluir en la ausencia de cualquier criterio YEARS y un DD $<1.0 \mu \mathrm{g} / \mathrm{m}^{40}$.

\section{FILTRO DE VENA CAVA EN EL EMBARAZO}

Desde la colocación del primer FVC en una paciente embarazada en 1981, el uso de esta técnica en pacientes seleccionadas se ha incrementado de forma progresiva, al principio con filtros permanentes y actualmente con los extraíbles ${ }^{35,36}$.

\section{INDICACIONES}

Mujeres pretérmino, cercanas al momento del parto o con alto riesgo de parto pretérmino y que requieren altas dosis de heparina por padecer una complicación tromboembólica o que presentan factores de riesgo de sangrado son candidatas a la implantación de un FVC extraíble. Las principales indicaciones para la colocación de un FVC se exponen en la Tabla 3. No todos los escenarios tienen el mismo grado de aceptación, de manera que la decisión sobre la colocación de un FVC debe ser cuidadosamente individualizada. En la tabla se incluyen las indicaciones más comúnmente aceptadas, así como otras descritas de forma esporádica, recogidas en la literatura. Si bien las indicaciones profilácticas no están claramente definidas, algunos autores han planteado su implantación en pacientes con trombofilias de alto riesgo, en el periparto ${ }^{43}$, mientras que otros limitan su uso a casos aislados y raros dónde la anticoagulación prolongada esté absolutamente contraindicada, no sólo durante el periodo del parto ${ }^{44}$.

Tabla 3. Situaciones clínicas en las que se ha comunicado la colocación de un FVC en mujeres embarazadas con evento tromboembólico asociado.

\begin{tabular}{|l|}
\hline Necesidad de altas dosis de heparina \\
\hline Cesárea programada \\
\hline Hipervolemia uterina \\
\hline Multiparidad \\
\hline Anomalías de inserción de la placenta \\
\hline Alta probabilidad de distocia fetal \\
\hline Necesidad de apoyo instrumental para el parto \\
\hline Cesárea urgente \\
\hline Trombocitopenia grave inducida por heparinas \\
\hline Alergia a la heparina \\
\hline Contraindicación para la anticoagulación (p.ej. neurocirugía reciente...) \\
\hline Presencia de trombo grande, inestable o flotante \\
\hline $\begin{array}{l}\text { Riesgo de embolia pulmonar ante procedimientos endovasculares } 0 \\
\text { trombólisis }\end{array}$ \\
\hline
\end{tabular}

\section{COLOCACIÓN}

Los FVC se han colocado con éxito tanto en primíparas como en multíparas, en todos los trimestres del embarazo, retirándose tras el parto 0 dejándose de forma semipermanente para futuros embarazos ${ }^{20,45,46}$. Las vías de inserción que se usan son la yugular 
y la femoral (más habitual) ${ }^{42,47}$. Si bien la información relativa al lugar idóneo de colocación del filtro es escasa, actualmente existe una preferencia por su colocación a nivel de la salida de las venas suprarrenales desde la vena cava inferior porque posteriormente a este nivel, el FVC puede ser comprimido por el útero grávido y favorecer así su desplazamiento o fractura pudiendo además dañar la pared de la vena cava inferior, teniendo en cuenta que la vena suprarrenal izquierda drena en la vena renal ipsilateral y la vena suprarrenal derecha directamente en la vena cava inferior ${ }^{19}$. La colocación suprarrenal protege además de los trombos que se puedan desarrollar en las venas ováricas dilatadas y no se ha documentado disfunción renal asociada ${ }^{19,48}$.

\section{EFECTIVIDAD}

Tras la colocación de un FVC extraíble, no se han comunicado embolismos pulmonares con resultado de muerte. La tasa de embolismos sintomáticos es baja, en torno al $0,81 \%{ }^{35}$. Con el advenimiento de los FVC extraíbles o recuperables, se ha visto que existen restos de trombo retenidos en muchos de ellos, pero no es posible saber si esos restos han podido provocar embolismos clínicamente significativos ${ }^{49}$.

\section{MORBIMORTALIDAD FETAL}

Los resultados de morbilidad y mortalidad en recién nacidos de madres portadoras de FVC son muy buenos y no se han descrito casos de muerte perinatal asociada. Se han comunicado dos casos de distrés respiratorio leve y hay información limitada sobre la evolución de estos niños a largo plazo ${ }^{31,50}$. Existe incertidumbre sobre cómo puede afectar el FVC en el embarazo al futuro del niño, motivada por la radiación que se debe de administrar para la colocación de dicho filtro. La International Commissionon Radiological Protection dice en sus recomendaciones que no cabe esperar efectos significativos que afecten al desarrollo fetal con dosis menores de $100 \mathrm{mGy}$. Las dosis habitualmente usadas varían entre 0,5 y 12 Gy20,51,52. Para minimizar esta cantidad de radiación que recibe el feto se usan protecciones abdominales de plomo, ecografía para diagnosticar la trombosis venosa profunda y colocación intravascular guiada por ecografía. Sin embargo, hasta el momento, para conocer exactamente dónde se quiere dejar colocado el filtro, se debe usar la fluoroscopia con la consiguiente radiación asociada ${ }^{20,53}$. Hara et al. estudiaron retrospectivamente a 11 mujeres con ETV durante el embarazo a las que se colocó un FVC sin evidenciar mortalidad perinatal asociada ${ }^{32}$.

\section{MORBIMORTALIDAD MATERNA}

En 1990 una madre falleció tras la colocación de un FVC, modelo Greenfield, en el segundo mes de gestación durante su colocación a la vena yugular interna ${ }^{54}$. Actualmente ya no se usa este filtro y las técnicas percutáneas de inserción femoral con vainas protectoras más pequeñas son muy seguras en este aspecto. Sally et al, en un metaanálisis ha encontrado una incidencia de complicaciones para la madre del $8,87 \%$ llegando al 11,3\% al incluir los casos de mortalidad o precipitación del parto. En este estudio, 80 pacientes portaban un filtro extraíble. En 8 casos no se pudo recuperar, en un caso la recuperación fue sólo parcial y en otro caso el filtro recuperado tuvo que ser reemplazado por uno permanente al presentar un trombo de gran tamaño que no se resolvió tras trombólisis guiada por catéter. Esto arroja una tasa de fracasos en la retirada de los filtros del 12,1\%, similar a poblaciones no embarazadas ${ }^{35}$.

Se han descrito casos de precipitación del trabajo del parto tras la colocación del FVC, incrementos transitorios en la actividad uterina, dolor e hinchazón en la zona de inserción femoral, infección del filtro en el parto como resultado de una rotura prolongada de membranas u otras causas de sepsis periparto ${ }^{41,55,56}$.

En el estudio con un seguimiento mayor de pacientes con filtro permanente, 18 pacientes durante 78 meses, no se comunicaron embolismos pulmonares o complicaciones relacionadas directamente con el filtro, pero sí una mayor frecuencia de hinchazón en las piernas probablemente en relación con la resolución parcial de la trombosis venosa profunda ${ }^{14}$.

Como ya se ha dicho, el embolismo pulmonar es la causa más frecuente de mortalidad materna en los países desarrollados. Detrás de ello se encuentran aspectos como el retraso diagnóstico y terapéutico, así como el uso de dosis inadecuadas de anticoagulantes en el tratamiento o la profilaxis tromboembólica. Un estudio retrospectivo no mostró mortalidad materna en un grupo de mujeres a las que se implantó un FVC extraíble durante el embarazo ${ }^{32}$.

Po último, la mayoría de las mujeres que tienen una trombosis durante el embarazo van a desarrollar secuelas como edema, cambios cutáneos, trombosis recurrente o desarrollo de úlceras².

\section{EL MOMENTO DEL PARTO}

La mujer que ha sufrido un evento tromboembólico durante el embarazo y se encuentra en tratamiento anticoagulante, tiene un mayor riesgo de sangrado durante el parto, tanto relacionado con la hemorragia del trabajo del parto como con la asociada a la realización de una cesárea. Las distintas opciones llegado el momento del parto incluyen el parto espontáneo no planeado, el parto planeado o la realización de una cesárea. La elección entre las opciones referidas se encuentra muy influenciada por las preferencias y experiencia del equipo que va a atender a la parturienta ${ }^{57}$. En general, llegado el momento del parto, muchos autores prefieren que la recomendación del método a seguir se base en criterios únicamente obstétricos ${ }^{45}$, mientras que otros recomiendan la realización de una cesárea porque conlleva un menor tiempo sin anticoagulación y, además, el parto vaginal se puede ver dificultado por la presencia de hinchazón en los miembros inferiores en las pacientes portadoras de un FVC ${ }^{20}$. Se ha especulado con la posibilidad de que las contracciones durante el trabajo del parto pudiesen favorecer la migración, ruptura 0 inclinación del filtro ${ }^{58}$.

\section{COMPLICACIONES ASOCIADAS}

Si bien la experiencia en la colocación de FVC en el embarazo es limitada, se han comunicado casos de complicación grave como rotura del filtro, migración, perforación de la vena cava o fallos en la retirada de filtros extraíbles ${ }^{45,58,59}$. En los casos donde no se puede retirar el filtro, probablemente se deba mantener el tratamiento anticoagulante de forma indefinida si bien no existe consenso actual a este respecto ${ }^{33,34}$.Las complicaciones más frecuentes asociadas con el uso del FVC durante el embarazo se muestran en la Tabla 4.

Tabla 4. Complicaciones asociadas a la colocación del FVC durante el embarazo.

\begin{tabular}{|l|}
\hline Rotura del filtro \\
\hline Migración del filtro \\
\hline Exposición fetal a la radiación \\
\hline Embolismo pulmonar tras la retirada del filtro \\
\hline Oclusión \\
\hline Embolización tras ruptura \\
\hline
\end{tabular}




\section{VALORACIÓN DE LA RETIRADA DEL FILTRO}

El hecho de poder usar en la actualidad filtros extraíbles ha contribuido a un incremento en su uso. Sin embargo, en muchas ocasiones no se retiran (12\%-45\%) por diversas razones. La posibilidad de padecer una complicación aumenta tras los primeros 30 días de su inserción y la mediana de retirada del FVC es de 72 días ${ }^{60}$.

Las recomendaciones actuales son la limitación del uso de FVC en mujeres gestantes con ETV a los casos de muy alto riesgo de recurrencia y retirarlos durante el postparto tan pronto como sea posible ${ }^{61}$.

\section{DISCUSIÓN Y CONCLUSIONES}

Actualmente, la colocación de un FVC temporal durante el embarazo es una alternativa útil y eficaz para el tratamiento de mujeres con alto riesgo de embolia pulmonar en las que el tratamiento anticoagulante no es eficaz por progresión de la trombosis o que presentan sangrado importante y trombocitopenia. Además, tras la colocación del FVC se puede reducir la dosis de heparina con la consiguiente reducción del riesgo potencial de mortalidad asociado al sangrado $0^{62,63}$.

El conocimiento relativo al beneficio de la colocación del FVC durante el embarazo con muy alto riego de embolismo pulmonar es limitado pues proviene fundamentalmente de casos clínicos individuales o series de pocos casos. Sin embargo, el balance riesgo beneficio que siempre tiene que realizar todo clínico a la hora de tratar a un paciente, parece indicar que la colocación de un FVC sea una opción a considerar en casos escogidos: mujeres embarazadas seleccionadas como aquéllas que requieren altas dosis de anticoagulación tras haber sufrido un evento tromboembólico en el peripato, con alto riesgo de parto prematuro, las que presentan complicaciones asociadas al uso del tratamiento anticoagulante 0 con un mayor riesgo de sangrado obstétrico durante el parto ${ }^{44,63}$.

En mujeres embarazadas que, tras sufrir un evento tromboembólico, precisan altas dosis de tratamiento anticoagulante, con mayor riesgo de sufrir sangrado asociado al tratamiento con heparina en el periparto o que presentan complicaciones asociadas el tratamiento con heparina, sería aconsejable poder reducir de alguna manera las dosis de heparina utilizadas. En estos casos, la colocación de un FVC podría ser una opción eficaz para prevenir la embolia pulmonar ${ }^{63}$. La realización de ensayos clínicos aleatorizados ayudaría sin duda a determinar la eficacia de este método terapéutico, así como sus efectos a largo plazo sobre madres e hijos.

Actualmente no existe evidencia a favor del uso rutinario del FVC en mujeres embarazadas con ETV. La decisión de usar un FVC durante el embarazo debe ser cuidadosamente valorada por un equipo multidisciplinar que estudiará de forma individualizada la relación riesgo/beneficio de su colocación.

\section{CONFLICTOS DE INTERESES}

Ios autores no declaran conflictos de intereses

\section{FINANCIACIÓN}

el estudio no ha recibido financiación externa

\section{CONSENTIMIENTOS ÉTICOS}

debido a las características del estudio no ha sido necesario solicitar consentimiento del Comité Ético.

\section{AGRADECIMIENTOS}

Los autores desean expresar su agradecimiento a la Dra. Ángeles Blanco Molina, Coordinadora del Grupo de Enfermedad Tromboembólica de la Sociedad Española de Medicina Interna por sus comentarios críticos del manuscrito.

\section{BIBLIOGRAFÍA}

1. Macklon NS, Greer IA. The deep venous system in the puerperium: an ultrasound study. Br J ObstetGynaecol. 1997; 104:198-200.

2. James AH, Tapson VF, Goldhaber SZ. Thrombosis during pregnancy and the postpartum period. Am J Obstet Gynecol. 2005; 193:216-219.

3. Marik PE, Plante LA. Venous thromboembolic disease and pregnancy. N Engl J Med. 2008; 359:2025-2033.

4. Heit JA, Kobbervig CE, James AH, Petterson TM, Bailey KR, Melton LJ 3rd. Trends in the incidence of venous thromboembolism during pregnancy or postpartum: a 30-year population-based study. Ann Intern Med. 2005; 143:697-706.

5. James $A H$, Jamison MG, Brancazio LR, Myers ER. Venous thromboembolism during pregnancy and the postpartum period: incidence, risk factors, and mortality. Am J Obstet Gynecol. 2006; 194:1311-1315.

6. Chang J, Elam-Evans LD, Berg CJ, Herndon J, Flowers L, Seed KA, Syverson CJ.Pregnancy-related mortality surveillance--United States, 1991--1999.MMWR SurveillSumm. 2003;52:1-8

7. McColl MD, Ellison J, Greer IA, Tait RC, Walker ID.Prevalence of the post-thrombotic syndrome in young women with previous venous thromboembolism. Br J Haematol. 2000; 108:272-274.

8. Rosfors S, Norén A, Hjertberg R, Persson L, Lillthors K, Törngren S.A 16-year haemodynamic follow-up of women with pregnancy-related medically treated iliofemoral deep venous thrombosis. Eur J VascEndovasc Surg. 2001; 22:448-455.

9. Macklon NS, Greer IA, Bowman AW. An ultrasound study of gestational and postural changes in the deep venous system of the leg in pregnancy. Br J ObstetGynaecol. 1997; 104:191-197.

10. Drife J.Thromboembolism. Reducing maternal death and disability during pregnancy. Br Med Bull. 2003;67:177-190.

11. Kujovich JL. Hormones and pregnancy: thromboembolic risks for women. $\mathrm{Br} \mathrm{J}$ Haematol. 2004; 126:443-454.

12. Eichinger S. D-dimer testing in pregnancy. Semin Vasc Med. 2005; 5:375-378.

13. Pomp ER, Lenselink AM, Rosendaal FR, Doggen CJ.Pregnancy, the postpartum period and prothrombotic defects: risk of venous thrombosis in the MEGA study. $J$ ThrombHaemost. 2008;6:632-637.

14. Aburahma AF, Mullins DA.Endovascular caval interruption in pregnant patients with deep vein thrombosis of the lower extremity.J Vasc Surg. 2001; 33:375-378.

15. Cheung MC, Asch MR, Gandhi S, Kingdom JC. Temporary inferior vena caval filter use in pregnancy. J ThrombHaemost. 2005;3:1096-1097.

16. Hynek K, Spálová I, Spatenka J, Mates M. Possibilities of using vena cava filters in pregnant women with venous thromboembolism. Case reports. Sb Lek. 2002; 103:451-454

17. Reddy K, Reginald PW, Charig MJ. Insertion of an inferior venocaval filter in a pregnant woman at risk for pulmonary embolism - a challenging management. J ObstetGynaecol. 2003; 23:198.

18. loakeimidou C, Aziz Al. To filter or not to filter the inferior vena cava in pregnancy? Case report. J ObstetGynaecol. 2005; 25:310.

19. Kawamata K, Chiba Y, Tanaka R, Higashi M, Nishigami K. Experience of temporary inferior vena cava filters inserted in the perinatal period to prevent pulmonary embolism in pregnant women with deep vein thrombosis. J Vasc Surg. 2005; 41:652-656.

20. Liu Y, Sun Y, Zhang S, Jin X. Placement of a retrievable inferior vena cava filter for deep venous thrombosis in term pregnancy. J Vasc Surg. 2012; 55:1042-1047.

21. Nakajima Y, Masaoka N, Tsuzuki Y, Morooka M, Sakai M. May-Thurner syndrome resulting in acute iliofemoral deep vein thrombosis during the second trimester of pregnancy. J ObstetGynaecol Res. 2012; 38:1106-1110.

22. Robertson L, Wu O, Langhorne P, Twaddle S, Clark P, Lowe GD, Walker ID, Greaves M, Brenkel I, Regan L, Greer IA. Thrombosis: Risk and Economic Assessment of Thrombophilia Screening (TREATS) Study.Thrombophilia in pregnancy: a systematic review.Br J Haematol. 2006;132:171-196.

23. De Stefano V, Rossi E, Za T, Leone G. Prophylaxis and treatment of venous thromboembolism in individuals with inherited thrombophilia. Semin ThrombHemost. 2006; 32:767-780.

24. Devis $P$, Knuttinen MG. Deep venous thrombosis in pregnancy: incidence, pathogenesis and endovascular management. Cardiovasc Diagn Ther 2017;7(Suppl 3): S309-S319.

25. Bates SM, Middeldorp S, Rodger M, James AH, Greer I. Guidance for the treatment and prevention of obstetric-associated venous thromboembolism. J Thromb Thrombolysis. 2016; 41:92-128.

26. Dargaud Y, Rugeri L, Fleury C, Battie C, Gaucherand P, Huissoud C, Rudigoz RC, Desmurs-Clavel H, Ninet J, Trzeciak MC. Personalized thromboprophylaxis using a risk score for the management of pregnancies with high risk of thrombosis: a prospective clinical study. J ThrombHaemost. 2017; 15:897-906.

27. Pettilä V, Leinonen P, Markkola A, Hiilesmaa V, Kaaja R. Postpartum bone mineral density in women treated for thromboprophylaxis with unfractionated heparin or LMW heparin.ThrombHaemost. 2002; 87:182-186.

28. Martel N, Lee J, Wells PS. Risk for heparin-induced thrombocytopenia with unfractionated and low-molecular-weight heparin thromboprophylaxis: a metaanalysis.Blood. 2005; 106:2710-2715 
29. Bates SM, Rajasekhar A, Middeldorp S, McLintock C, Rodger MA, James AH, Vazquez SR, Greer IA, Riva JJ, Bhatt M, Schwab N, Barrett D, LaHaye A, Rochwerg B.American Society of Hematology 2018 guidelines for management of venous thromboembolism: venous thromboembolism in the context of pregnancy.Blood Adv. 2018; 2:3317-3359.

30. Hall JG, Pauli RM, Wilson KM. Maternal and fetal sequelae of anticoagulation during pregnancy. Am J Med. 1980; 68:122-140.

31. González-Mesa E, Azumendi P, Marsac A, Armenteros A, Molina N, Narbona I, Herrera J, Artero I, Rodríguez-Mesa JM.Use of a temporary inferior vena cava filter during pregnancy in patients with thromboembolic events. J ObstetGynaecol. 2015; 35:771-776.

32. Hara N, Miyamoto T, Yamaguchi J, Iwai T, Hijikata S, Watanabe K, Sagawa Y, Masuda R, Miyazaki R, Miwa N, Sekigawa M, Yamaguchi T, Nagata Y, Nozato T, Kobayashi 0, Umezawa S, Obayashi T. Treatment Outcomes of Anticoagulant Therapy and Temporary Inferior Vena Cava Filter Implantation for Pregnancy Complicated by Venous Thrombosis. Ann Vasc Dis. 2018; 11:106-111.

33. Crosby DA, Ryan K, McEniff N, Dicker P, Regan C, Lynch C, Byrne B. Retrievable Inferior vena cava filters in pregnancy: Risk versus benefit? Eur $\mathrm{J}$ ObstetGynecolReprod Biol. 2018; 222:25-30.

34. Rottenstreich A, Kalish Y, Elchalal U, Klimov A, Bloom Al. Retrievable inferior vena cava filter utilization in obstetric patients. J Matern Fetal Neonatal Med. 2019; 32:3045-3053.

35. Harris SA, Velineni R, Davies AH.Inferior Vena Cava Filters in Pregnancy: A Systematic Review.J VascIntervRadiol. 2016; 27:354-360.

36. Torkzad MR, Bremme K, Hellgren M, Eriksson MJ, Hagman A, Jörgensen T, Lund K, Sandgren G, Blomqvist L, Kälebo P. Magnetic resonance imaging and ultrasonography in diagnosis of pelvic vein thrombosis during pregnancy. Thromb Res. 2010; 126:107-112.

37. Konstantinides SV, Meyer G, Becattini C, Bueno H, Geersing GJ, Harjola VP, Huisman MV, Humbert M, Jennings CS, Jiménez D, Kucher N, Lang IM, Lankeit M, Lorusso R, Mazzolai L, Meneveau N, Áinle FN, Prandoni P, Pruszczyk P, Righini M, Torbicki A, Van Belle E, Zamorano JL; The Task Force for the diagnosis and management of acute pulmonary embolism of the European Society of Cardiology (ESC).2019 ESC Guidelines for the diagnosis and management of acute pulmonary embolism developed in collaboration with the European Respiratory Society (ERS): The Task Force for the diagnosis and management of acute pulmonary embolism of the European Society of Cardiology (ESC).Eur Respir J. 2019;54:1901647.

38. Righini M, Robert-Ebadi H, Elias A, Sanchez O, Le Moigne E, Schmidt J, Le Gall C, Cornuz J, Aujesky D, Roy PM, Chauleur C, Rutschmann OT, Poletti PA, Le Gal G; CT-PE-Pregnancy Group.Diagnosis of Pulmonary Embolism During Pregnancy: A Multicenter Prospective Management Outcome Study.Ann Intern Med. 2018;169:766-773.

39. Van der Hulle T, Cheung WY, Kooij S, Beenen LFM, van Bemmel T, van Es J, Faber LM, Hazelaar GM, Heringhaus C, Hofstee H, Hovens MMC, Kaasjager KAH, van Klink RCJ, Kruip MJHA, Loeffen RF, Mairuhu ATA, Middeldorp S, Nijkeuter M, van der Pol LM, Schol-Gelok S, Ten Wolde M, Klok FA, Huisman MV; YEARS study group.Simplified diagnostic management of suspected pulmonary embolism (the YEARS study): a prospective, multicentre, cohort study.Lancet. 2017;390:289-297.

40. Van der Pol LM, Tromeur C, Bistervels IM, Ni Ainle F, van Bemmel T, Bertoletti $L$, Couturaud F, van Dooren YPA, Elias A, Faber LM, Hofstee HMA, van der Hulle T, Kruip MJHA, Maignan M, Mairuhu ATA, Middeldorp S, Nijkeuter M, Roy PM, Sanchez O, Schmidt J, Ten Wolde M, Klok FA, Huisman MV; Artemis Study Investigators.Pregnancy-Adapted YEARS Algorithm for Diagnosis of Suspected Pulmonary Embolism.N Engl J Med. 2019;380:1139-1149.

41. Scurr J, Stannard $P$, Wright J. Extensive thrombo-embolic disease in pregnancy treated with a Kimray Greenfield vena cava filter. Case report. Br J ObstetGynaecol. 1981; 88:778-780

42. Neill AM, Appleton DS, Richards P. Retrievable inferior vena caval filter for thromboembolic disease in pregnancy. Br J ObstetGynaecol. 1997; 104:14161418.
43. Hidaka N, Hachisuga M, Tsukimori K, Onohara T, Maehara Y, Wake N. Intrapartum placement of an inferior vena cava filter for a woman with hereditary antithrombin III deficiency: its role in the prevention of fatal pulmonary embolism. J ObstetGynaecol Res. 2008; 34:95-99.

44. WiegersH, MiddeldorpS. Contemporary best practice in the management of pulmonary embolism during pregnancy. Ther Adv Respir Dis. 2020; 14:1-20.

45. Gupta S, Ettles DF, Robinson GJ, Lindow SW. Inferior vena cava filter use in pregnancy: preliminary experience. BJOG. 2008; 115:785-788.

46. Thomas LA, Summers RR, Cardwell MS. Use of Greenfield filters in pregnant women at risk for pulmonary embolism. South Med J. 1997; 90:215-217.

47. Narayan H, Cullimore J, Krarup K, Thurston H, Macvicar J, Bolia A. Experience with the Cardial inferior vena cava filter as prophylaxis against pulmonary embolism in pregnant women with extensive deep venous thrombosis. $\mathrm{Br} \mathrm{J}$ ObstetGynaecol. 1992; 99:637-640.

48. Greenfield LJ, Proctor MC. Suprarenal filter placement. J Vasc Surg. 1998; 28:432438

49. Neri E, Civeli L, Benvenuti A, Toscano T, Miraldi F, Capannini G, Muzzi L, Sassi C. Protected iliofemoral venous thrombectomy in a pregnant woman with pulmonary embolism and ischemic venous thrombosis. Tex Heart Inst J. 2002; 29:130-132.

50. Hux CH, Wapner RJ, Chayen B, Rattan P, Jarrell B, Greenfield L. Use of the Greenfield filter for thromboembolic disease in pregnancy. Am J Obstet Gynecol. 1986; 155:734-737.

51. Valadares S, Serrano F, Torres R, Borges A. Inferior Vena Cava Filter Placement during Pregnancy: An Adjuvant Option When Medical Therapy Fails. Case Rep Obstet Gynecol. 2013;2013:821635.

52. International Commission on Radiological Protection. The 2007 recommendations of the International Commission on Radiological Protection. ICRP Publication 103. Ann ICRP. 2007; 37:1-332.

53. Hodgkiss-Harlow K, Back MR, Brumberg R, Armstrong P, Shames M, Johnson B, Bandyk DF. Technical factors affecting the accuracy of bedside IVC filter placement using intravascular ultrasound. Vasc Endovascular Surg. 2012; 46:293-299.

54. Eagle CJ, Davies JM. Lethal air embolism during placement of a Kimray-Greenfield filter. J CardiothoracAnesth. 1990; 4:616-620

55. Quayle S, Germain M, Wolf E, Miller R. Case report of placement of a bird's nest filter during pregnancy. J Matern Fetal Med. 1994: 3:187-189.

56. Barnes AB, Kanarek DJ, Greenfield AJ Jr, Brewster DC. Vena caval filter placement during pregnancy. Am J Obstet Gynecol. 1981; 140:707-708.

57.-Middeldorp S. How I treat pregnancy-related venous thromboembolism. Blood. $2011 ; 118: 5394-5400$

58. Ganguli S, Tham JC, Komlos F, Rabkin DJ. Fracture and migration of a suprarenal inferior vena cava filter in a pregnant patient. J VasclntervRadiol. 2006; 17:17071711.

59. McConville RM, Kennedy PT, Collins AJ, Ellis PK. Failed retrieval of an inferior vena cava filter during pregnancy because of filter tilt: report of two cases. Cardiovasc InterventRadiol. 2009; 32:174-177.

60. Angel LF, Tapson V, Galgon RE, Restrepo MI, Kaufman J. Systematic review of the use of retrievable inferior vena cava filters. J VascIntervRadiol. 2011; 22:15221530.e3.

61. Bates SM, Greer IA, Middeldorp S, Veenstra DL, Prabulos AM, Vandvik PO. VTE, thrombophilia, antithrombotic therapy, and pregnancy: Antithrombotic Therapy and Prevention of Thrombosis, 9th ed: American College of Chest Physicians EvidenceBased Clinical Practice Guidelines. Chest. 2012; 141(2 Suppl): e691S-e736S.

62. Duffett L, Carrier M. Inferior vena cava filters. J ThrombHaemost. 2017; 15:3-12.

63. Fogerty AE. Management of Venous Thromboembolism in Pregnancy. Curr Treat Options Cardiovasc Med. 2018; 20:69 